\title{
Empresa, crisis y educación universitaria en el Perú
}

\author{
Jenny Canales Peña \\ Universidad Antonio Ruiz de Montoya
}

\section{Resumen}

En el actual sistema universitario, existen más de cien universidades, mayoritariamente privadas, lo cual convierte al sistema en uno empresarial. Así, el interés por obtener una porción del mercado hace que se pierda de vista la esencia de la universidad, el lugar que ocupa el docente y la vida universitaria, y reducen las expectativas académicas a unas práctico-utilitarias. Se exige al docente que capte o mantenga al estudiantado, pero no se le permite la pertenencia a un centro ni al sistema en general. La crisis evidencia una despreocupación por aspectos antropológicos; la persona no tiene un espacio en el sistema liderado por la economía.

Palabras clave: universidad, empresa, docente universitario

\begin{abstract}
In the actual Peruvian university system, they exist more than one hundred universities, most lf them private, which turns it into a business system. That way, the interest for obtaining a piece of the market make the essence of the university get lost of sight, as well as the place that occupy the professor and the university life, and they reduce the academical expectations to practical utilitarian. It is required that the professors keep the students, but the membership to a center nor at the general system is not allowed. The crisis shows an unconcern for anthropological aspects; the people don't have a space in the economy ruled system.
\end{abstract}

Key words: university, company, university professor 


\section{Introducción}

Desde el siglo pasado, se dice que la universidad está en crisis y lo afirmaba el Dr. Alberto Tauro del Pino en el discurso en la Ceremonia de Imposición de Insignias de Profesor Emérito de la Universidad Nacional Mayor de San Marcos, en agosto de 1986, aludiendo a que la crisis era el reflejo de lo que sucedía en otras instituciones del país y que se propiciaba por las contradicciones y los cambios en la sociedad peruana. Vale agregar que la universidad pública siempre es el referente de Universidad en el país.

Así, se han realizado distintas acciones para salir de la crisis, se han dado múltiples reformas a la ley universitaria ${ }^{1}$, se han creado organismos de control$^{2}$, se ha apostado por la construcción de un

1 En el siglo $\mathrm{xx}$, existieron, al menos, seis reformas universitarias que cambian pequeños aspectos de la anterior. La primera se inició en el gobierno de Leguía (1928) con el Estatuto Universitario; tres ańos después, se realiza la ley de Reforma Universitaria, en el gobierno de Sánchez Cerro; en 1935, se volvió a la primera ley, en el gobierno de Benavides; en el gobierno de Bustamante y Rivero (1945), se promulgó la ley No 10555; en 1960, el segundo gobierno de Prado, se emitió la ley No 13417 que marca el nacimiento de varias universidades en el interior del país. En 1983, en el gobierno de Belaúnde, se promulgó la ley universitaria No 23733 y se creó la Asociación Nacional de Rectores -ANR, en estos momentos se declaran 26 universidades nacionales y nueve privadas y, en 1996, con el Decreto Legislativo No. 882 se promulga la ley de Promoción de Inversión en Educación y se crea el Consejo Nacional para la Autorización y el Funcionamiento de Universidades -CONAFU, en el gobierno de Fujimori. Recuperado de: <http://www.losandes. com.pe/oweb/educacion/20140504/79956.html>

2 En el 2014 se crea la Superintendencia Nacional de Educación Superior Universitaria -SUNEDU- como organismo público, técnico-especializado, adjunto al Ministerio de Educación con autonomía técnica, funcional, económica, presupuestal y administrativa, ejerce su jurisdicción a nivel nacional. La SUNEDU remplaza a la Asamblea Nacional de Rectores ANR y al CONAFU. En el Artículo 13 , respecto de su finalidad, plantea que es responsable del licenciamiento para el servicio educativo superior universitario, entendiéndose el licenciamiento como el procedimiento que tiene como objetivo verificar el cumplimiento de condiciones básicas de calidad para ofrecer el servicio educativo superior universitario y autorizar su funcionamiento. 
modelo de aseguramiento de la calidad ${ }^{3}$, se han adaptado los planes de estudios a las exigencias cambiantes del mercado, se ha considerado la capacitación de los y las docentes como una actividad válida y necesaria para brindar diferentes habilidades y conocimientos en el manejo de nuevas herramientas; incluso, desde el siglo xxI, se exige que para ejercer la docencia universitaria, el docente debe tener el grado de maestro, pero ¿estas acciones han sido suficientes para salir de la crisis? ¿Qué puede estar pasando? o ¿es que todavía no se ha identificado el verdadero problema de la crisis?

\section{Algunos breves antecedentes ${ }^{4}$}

La primera universidad nacional creada en el Perú data de 1551, a raíz de un alegato promovido por fray Tomás de San Martín, que consideró posible usar parte de las instalaciones del convento de Santo Domingo. Luego, en 1677, el obispo Don Cristóbal de Castilla y Zegarra, creó la Universidad San Cristóbal y Zamora en Huamanga (Ayacucho), aunque por razones económicas y por la oposición negativa de la Universidad de San Marcos, se clausuró ${ }^{5}$. Luego, en 1696, se crea la Universidad San Antonio Abad del Cusco y, más tarde, en la época Republicana, la Universidad Nacional de Trujillo (1824), la Nacional de San Agustín de Arequipa (1827) y la San Carlos de Puno (1856); todas funcionan con la estructura académica y administrativa de la Universidad Nacional Mayor de San Marcos.

Posteriormente, en 1854, se crea la Escuela Normal Central y, recién en el siglo XX, en el año 1917, inicia sus actividades, como

3 El Sistema Nacional de Evaluación, Acreditación y Certificación de la Calidad Educativa -SINEACE- es un organismo técnico-especializado, adscrito al Ministerio de Educación, creado en el 2006 a través de la Ley No 28740. Su finalidad es garantizar a la sociedad que las instituciones educativas públicas y privadas ofrezcan un servicio de calidad, y que los trabajadores peruanos estén altamente calificados para la labor que realizan.

4 Recuperado de: <https://cnmalpica.wordpress.com/2015/10/02/20150929-carlosmalpica-faustor-universidad-y-planes-de-gobierno-2016-2021-ponencia-foro-dela-camara-de-comercio-de-lima/>.

5 En 1957, se reabrió como Universidad de Ayacucho. 
Educación Superior Privada, la Universidad Católica del Perú por gestión del padre Jorge Dintilhac. Para 1979, existían en el país 19 universidades y cinco de ellas, privadas.

Antes de terminar el siglo xx, en 1996, se promulga la Ley de Promoción de la Inversión en la Educación ${ }^{6}$, que permite el incremento sustancial de universidades ${ }^{7}$ y, con ello, de matrícula de estudiantes, de personal administrativo y docente. La implantación del modelo, a decir de los sectores implicados, conseguiría ampliar la oferta y la cobertura universitaria; además, el acceso a los estudios superiores se democratizaría y así más jóvenes de todos los estratos económicos estudiarían en la universidad y, se supuso, que el mercado sería un regulador efectivo de la calidad de este servicio, mediante el cual la competencia dejaría fuera a las universidades de mala calidad (Cuenca, 2015).

\section{La crisis de la universidad en el siglo XXI}

Como afirma Cuenca, con el paso del tiempo, vemos que solamente lo primero se cumplió, pues al competir como empresa privada todos buscaban un mismo mercado estudiantil, con lo cual se trastocaron algunos aspectos vinculados a la vida universitaria. Así, se brindó mayor importancia al aspecto administrativo y sobre todo al comercial, que decidía qué es lo más "vendible" y los planes de estudio se adaptaron a ello; además, algunos decanatos o algunas direcciones de carrera fueron asumidos por buenos gestores que no tenían experiencia académica y esto afectó sensiblemente la labor del docente universitario y de la

6 El Decreto Legislativo 882: Ley de Promoción de la Inversión en la Educación, plantea en su artículo 2 lo siguiente: "Toda persona natural o jurídica tiene derecho a la libre iniciativa privada, para realizar actividades en la educación. Este derecho comprende los de fundar, promover, conducir y gestionar Instituciones Educativas Particulares, con o sin finalidad lucrativa.” Diario El Peruano, pág. 144-157, año XVI, Na 5975.

7 En el 2014, había 140 universidades en todo el país y de 255 mil matriculados que eran alrededor de los años 80, se alcanzó más de 900 mil en 2014. 
carrera ${ }^{8}$ hacia la docencia; ello sumado a que las regulaciones impartidas desde el Estado afectaban directamente a las universidades estatales y a su comunidad, y dejaron fuera a todos los otros que, aunque eran del mismo rubro académico, no pertenecían al sector económico; es decir, en este esquema, el docente universitario se convierte en un empleado que, ubicado después del área administrativa, tiene una relación temporal con la universidad, pero sin referencia a nivel -auxiliar, asociado o principal-, lo cual no le permite tener una carrera docente. La docencia universitaria se modifica, pues ya no se le vincula con lo académico, el docente no decide ${ }^{9}$, trabaja a destajo ${ }^{10}$, lo cual lo convierte en un invitado de turno obligado a buscar otros diferentes espacios laborales e implica que se pierde la comunidad académica, así como la producción y la difusión de investigación de modo integrado. La pertenencia a diferentes culturas organizativas de empresas universitarias distintas, lejos de unir, dista de lo que la Universidad -hablando en genéricoprecisa. Se trata, entonces, de una crisis de identidad planteada entre lo empresarial y lo universitario.

\section{La universidad y la vida universitaria}

Intentar que la concepción con la que se creó la universidad aún prevalezca es un poco ingenuo, pues las condiciones histórico-sociales y las necesidades del país son diferentes; sin embargo, no se puede olvidar

8 Antes del Decreto Legislativo 882, las universidades promovían la constitución de asistentes de cátedra, impartida por estudiantes que cumplían ciertos requisitos, así como la jefatura de práctica que, generalmente, la impartía el egresado o bachiller para luego acceder a una plaza docente.

9 Hasta antes de la creación de SUNEDU, en universidades creadas con la Ley de Promoción y Fomento no existe necesariamente el Consejo de facultad ni el Consejo universitario, ambos reemplazados por el directorio conformado por los inversionistas y el Rector, quien depende y rinde ante el presidente del directorio.

10 En muchas universidades privadas de Lima, el docente percibe remuneración solo si logra brindar la clase asignada; si es feriado o sufre algún percance, no, e incluso no siempre existe la posibilidad de recuperación de clase. Por otro lado, las contrataciones son a tiempo parcial, no más de 20 horas semanales y si se obtiene el tiempo completo, algunas solicitan hasta 20 horas de dictado de clase en aula. 
que, parafraseando al cardenal John Henry Newman, autor de The Idea of a University, la función primordial de la universidad consiste en trascender lo útil y dar espacio a la "educación liberal"11 en el campo social o económico. Como diría Portocarrero (2017), Esto es trascender la inutilidad.

La mayoría de las universidades surgidas en la modernidad no lograron entender claramente a qué se refiere el término 'liberal', pues ni se brindó educación general como sí se hizo en algunas universidades estadounidenses ${ }^{12}$, ni se consideró a las humanidades como un referente importante para el futuro profesional. El sistema utilizado consideró que lo más necesario era formar profesionales en el "hacer" más que en el "conocer", en el sentido amplio.

Aunque la empresa universitaria tampoco termina de comprender qué es lo que se debe conocer, Karl Jaspers, en La idea de universidad (2013), considera que el conocer es un fin en sí mismo, que la universidad plantea la exigencia del deseo de conocer sin consideraciones y ello solo es posible con la propia responsabilidad del individuo. Por su parte, Leonardo Polo (2018) agrega que la universidad aporta a la sociedad el saber superior, enfatiza que esta educación no es ni lo último en la escala de la formación ni es lo que le sigue a la educación secundaria, sino que la universidad brinda la posibilidad de incrementar permanentemente el saber -un crecimiento irrestricto-, en función de los cambios histórico-sociales.

11 Para Newman, la educación caracterizada como "liberal" se convertía en un hábito mental o en un hábito filosófico, pues duraba toda la vida y sus características eran freedom, equitableness, calmness, moderation and wisdom (Portocarrero, 2018, p. 139).

12 José Torralba, en un artículo publicado en Actas filosóficas al realizar un recuento de la educación en Estados Unidos, indica que la educación liberal está vinculada a la formación integral o general, cuyo objetivo es prevenir contra la concepción utilitaria del conocimiento, así como de las diversas formas de parcialidad y dogmatismos. Generalmente, son instituciones denominadas liberal Arts Colleges. (Acta Philosofica II, No 22, 2013) 
Por ello, aportar a la sociedad va mucho más allá de llenarla de profesionales ${ }^{13}$ o de dinamizar el mercado. La educación universitaria, como diría Newman, "forma "gentlemen" y sus características esenciales giran alrededor de cómo cultivar el intelecto, obtener un gusto refinado, desarrollar una mente sincera, desapasionada, justa, que ayude a construir una conducta de vida noble y cortés" (Portocarrero, 2017, p. 142).

La educación universitaria vincula a la persona con su convivencia, con el respeto al otro, con la posibilidad de crear ciudadanos, no meros ejecutores prácticos que solo pueden resolver problemas específicos de su entorno. Lo esencial es comprender al otro como individuo para crear una sociedad civil y una convivencia comunitaria.

Para Newman, la verdadera y más adecuada finalidad de una universidad no es el aprendizaje o la adquisición de conocimientos, sino el pensamiento y la razón ejercidos sobre el conocimiento (Portocarrero, 2017, p. 146).

Esto puede parecer poco útil o contrario a la economía, pero el universitario que logre ser crítico, que sepa discernir, podrá asumir cualquier profesión de manera libre, pues tendrá una expansión mental. De este modo, los conocimientos son solo un medio para lograr el saber superior que permita el bien común y, por ende, beneficie a la sociedad en su conjunto.

Y esto es parte de la vida universitaria que se ha perdido, el espacio para compartir, para discutir, para conocer y conocerse. Jaspers afirma que el proceso de comunicación, en la universidad, se inicia con el cuestionar. Sin embargo, la rapidez por terminar la carrera para ejercer en el mercado, para hacer que la inversión retorne, no permiten al docente ni al estudiante detenerse a reflexionar.

13 Lavado, Martínez y Yamada (2015), en el artículo "Efectos persistentes del subempleo profesional, 2004-2014. Evolución de subempleos en egresados de universidades e institutos técnicos", afirman que, aproximadamente, el $45 \%$ de las personas que tienen estudios superiores se encuentra trabajando en algo para lo cual no estudiaron, pp. 157-182. En Educación superior y empleo en el Perú: una brecha persistente (2017). 
Las empresas universitarias han ingresado a la vida universitaria asumiendo que cualquiera puede aprender y cualquiera enseńar; el estudiante es ahora un cliente, que busca avanzar, culminar su carrera y exige que se le muestre recetas de solución, y el docente es solo un transmisor, un dictante, un colaborador que, en el mejor de los casos, conseguirá que haya nuevos estudiantes. El director es un gestor que lidia con temas más vinculados al márketing que a lo académico.

Sin embargo, en la vida universitaria, el docente es, ante todo, un universitario y debe actuar de modo riguroso, disciplinado, con dedicación exclusiva, debe "intercambiar saber entre sus pares y discutir con sus estudiantes", como bien dice Polo, lo que se conoce como comunidad. De ahí, que para Polo el profesor universitario no puede ser un advenedizo, debe estar permanentemente preocupado por su enseñanza y por la creación de sucesores. El profesor es la dimensión de la universidad y por ello, él debe hacer suya la universidad (Polo, 2018).

La preocupación por la enseńanza empieza en el docente, pues es él quien primero aprende, el que asimila, el que organiza el conocimiento para impartirlo, no para instruir, sino para discutirlo con el otro, en tanto le despierta interés por aprender. Por eso, Polo habla de crear sucesores, ya que serán los estudiantes -los preocupados por el conocimiento- los que seguirán con la docencia universitaria.

De acuerdo con Leonardo Polo (2018), el docente en la universidad debe ser un provocador, pero uno que provoque descubrimientos trascendentales, que sea capaz de plantear grandes preguntas y que logre el incremento del saber. Debe ser un líder, comprendido como quien descubre lo que los otros son incapaces de ver [...] aprovechando una serie de potencialidades para desarrollarlas (Polo y Llano, 1997).

En este sistema empresarial, tampoco se le otorga valor a la comunidad docente, a la discusión entre pares, a la relación de nuevos y antiguos docentes; el diálogo ha perdido espacio y se ha olvidado que todos los saberes tienen que ver entre sí, que no son compartimientos independientes.

Las empresas, por lo general, determinan su identidad corporativa $y$, a partir de ello, construyen su mensaje publicitario, buscan propuestas 
que se asemejen con las necesidades percibidas. La empresa brinda -o es el ideal- clara orientación del camino, la ruta y las opciones que se ha de seguir para mejorar en calidad. La relación entre la identidad -lo que dice que es- y la imagen -lo que el otro cree que es- debe ser tan estrecha para que se incremente su reputación. Y, en este afán de calidad, capacitan a su personal en el uso de herramientas o de habilidades para satisfacer a los clientes, pero se olvida que el saber en la vida universitaria traspasa lo pedagógico, metodológico o tecnológico, pues al generar intercambio de conocimiento es preciso reforzar la sensibilidad, la generosidad, la comunicación, aspectos que le permitan conocer y reconocer a las personas, de tal modo que se pueda brindar y tener una formación humana y personal.

Actualmente, cada empresa universitaria es independiente, ha creado cada una su discurso -algunos más atractivos que otros- sin entender, como plantea Lerner, que la Universidad tiene como misión fundamental el formar personas con altas cualidades tanto científicas como humanas y que debe ser ella misma ejemplo de búsqueda permanente de calidad. Pero repárese que no debemos entender el término calidad como simple equivalente a una eficacia o eficiencia racionalizadas. Una cultura de calidad debe partir del hecho de que las personas tiendan a percibir su trabajo como un modo de vida y, dado que la calidad que persigue la universidad está constituida por la búsqueda de un desarrollo humano y social integral, es natural que esta se afiance en el natural deseo de toda persona de afirmarse humanamente como parte de una comunidad (Lerner, 2000, p. 12). La universidad no solo satisface al mercado, satisface a la comunidad.

La universidad es, junto a la familia y la empresa, como plantea Polo, un agente social y es una obligación urgente para quienes formamos para de ella comprender, como afirma Lerner que, la universidad antes que un diagnóstico debe ser un proyecto.

\section{Conclusiones}

El principal aspecto que se debe considerar es qué tipo de empresa requiere la educación universitaria y con ello qué tipo de personas deben de dirigirla, pues se sabe que si no se logra comprender la misión de la educación universitaria no se podrá avanzar como institución. 
Por otro lado, es importante reforzar el aspecto humano de quienes participan en la comunidad universitaria, pues el trabajo que se realiza es con y para personas, para la sociedad en su conjunto.

Es relevante comprender que, aunque el rol del docente universitario se ha modificado, ya que ahora no es el único portador del saber, es el docente quien se encuentra más cerca de los futuros profesionales; ello requiere unas condiciones antropológicas para ejercer la docencia.

Finalmente, las empresas de educación universitaria deben considerar realizar acciones conjuntas, de intercambio y de alianza, porque ello demostrará a nuestros estudiantes que en equipo se puede lograr el bien común.

\section{Referencias}

Cuenca, R. (edit.) (2015) La educación universitaria en el Perú. Democracia, extensión y desigualdades. Lima: IEP.

Jaspers, K. (2013) La idea de universidad. Navarra: Ediciones de la Universidad de Navarra, S.A.

Lerner, S. (2000) Reflexiones en torno a la universidad. Lima: Pontifica Universidad Católica del Perú.

Polo, L. (2018) Claves de la universidad y del profesor universitario. Navarra: Ediciones de la Universidad de Navarra, S.A.

Polo, L. y Llano, C. (1997) Antropología de la acción directiva. Madrid: Unión editorial S.A.

Portocarrero, F. (2017) La idea de universidad reexaminada y otros ensayos. Lima: Universidad del Pacífico. 
Tauro del Pino, A. (1986) Diorama de la crisis universitaria. Lima: Suplemento de la Gaceta sanmarquina, Año II, Na 2, cuarta época.

Torralba, J. (2015) La educación liberal como misión de la universidad. Introducción bibliográfica al debate sobre la identidad de la universidad. En Acta Philosophica II, No 22, pp. 257-276.

Yamada, G. y Lavado, P. (edit.) (2017). "Efectos persistentes del subempleo profesional, 2004-2014. Evolución de subempleos en egresados de universidades e institutos técnicos", En: Educación superior y empleo en el Perú: una brecha persistente. Lima: Universidad del Pacífico. 